\title{
Identification of Blanket design points using an integrated multi-physics approach
}

\section{G.A. Spagnuolo ${ }^{1}$, F. Franza ${ }^{1}$, U. Fischer ${ }^{1}$, L.V. Boccaccini ${ }^{1}$}

${ }^{1}$ Karlsruhe Institute of Technology, Institute for Neutron Physics and Reactor Technology (INR)

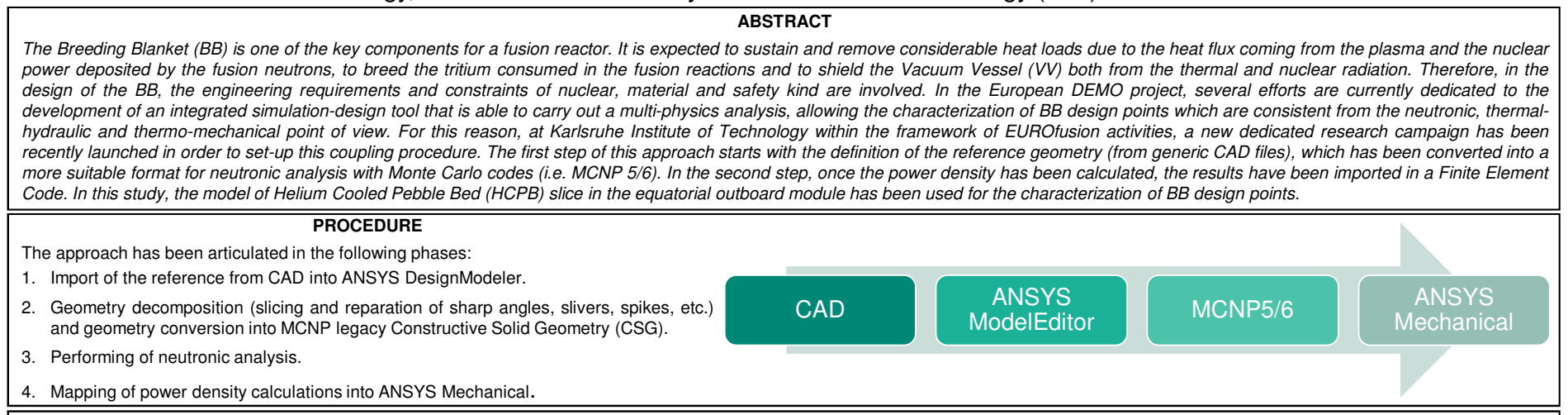

\section{Mapping of power density calculations into ANSYS Mechanical.}

GEOMETRY DECOMPOSITION

a It has been sliced in order to have simple configuration suitable for the CSG representation;

a The VV, the gap between the VV inner wall and the BB have been introduced;

- The cooling channels have been filled to model the coolant;

- The corners of the cooling channels have been squared;

a An enclosure has been used to shape the Graveyard.
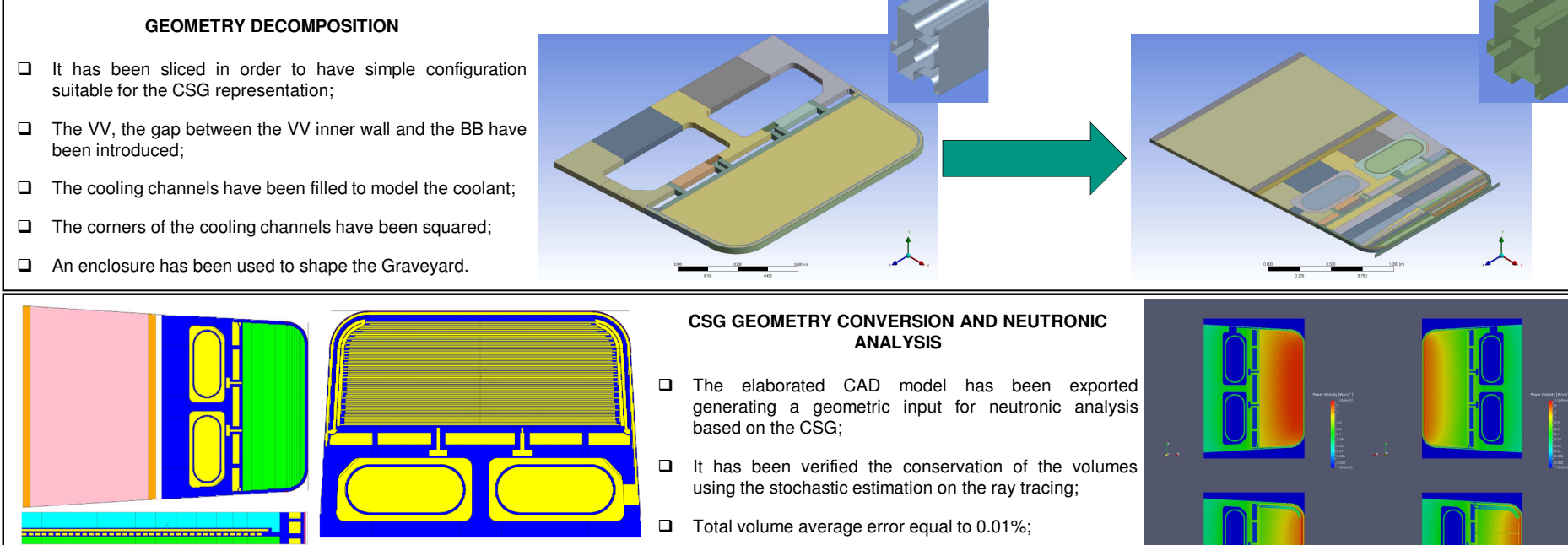

CSG GEOMETRY CONVERSION AND NEUTRONIC ANALYSIS
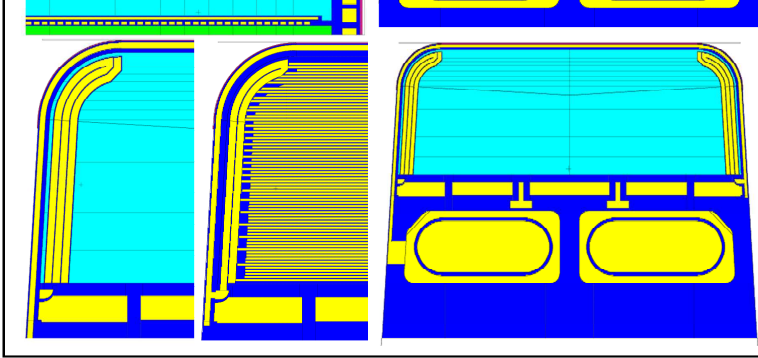

- The elaborated CAD model has been exported generating a geometric input for neutronic analysis based on the CSG;

口 It has been verified the conservation of the volumes using the stochastic estimation on the ray tracing;

a Total volume average error equal to $0.01 \%$;

口 Maximum volume error between $0.811 \%$ and $-0.725 \%$;

- It has been used a mono-energetic test source biased in the angular distribution;
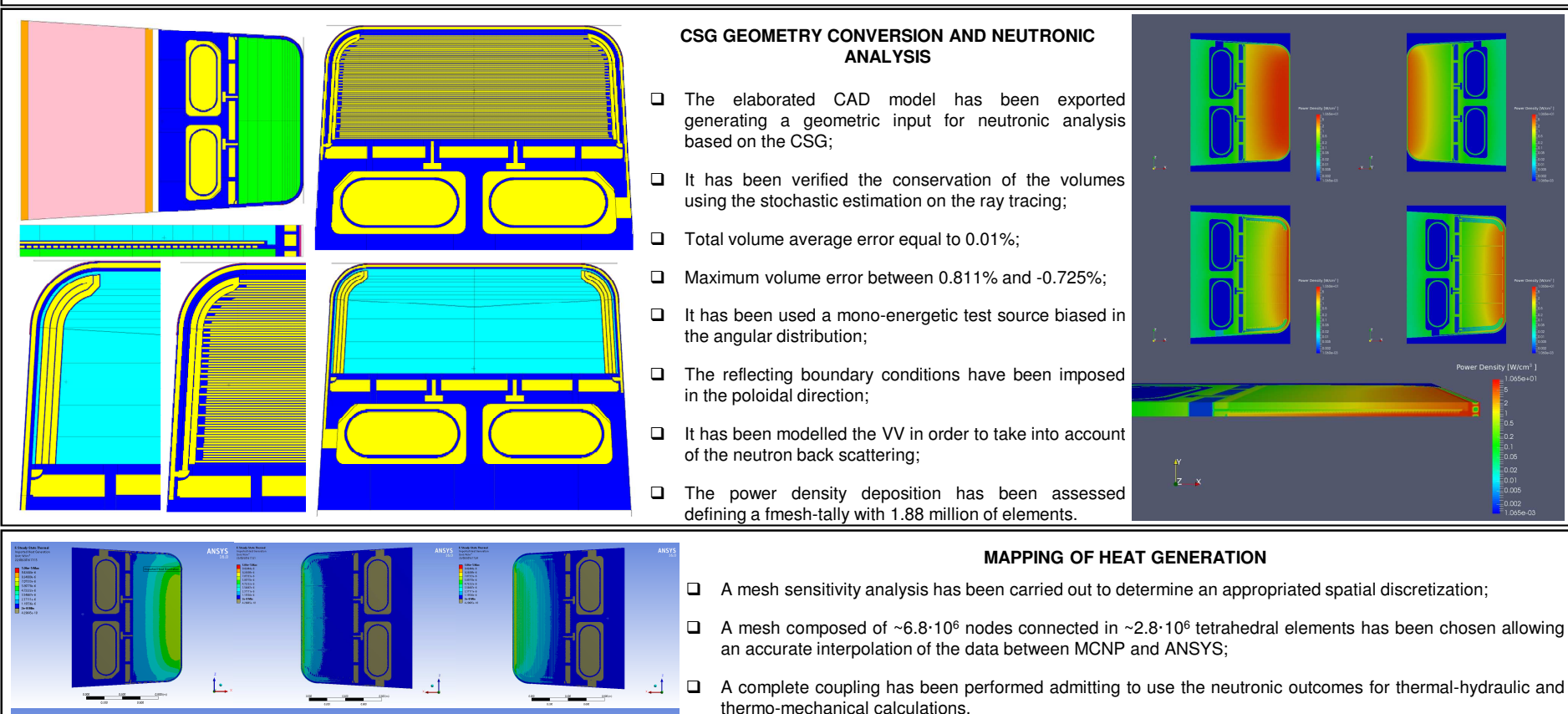

- The reflecting boundary conditions have been imposed in the poloidal direction;

- It has been modelled the VV in order to take into account of the neutron back scattering;

The power density deposition has been assessed defining a fmesh-tally with 1.88 million of elements.

\section{MAPPING OF HEAT GENERATION}

A mesh sensitivity analysis has been carried out to determine an appropriated spatial discretization:

a A mesh composed of $\sim 6.8 \cdot 10^{6}$ nodes connected in $\sim 2.8 \cdot 10^{6}$ tetrahedral elements has been chosen allowing an accurate interpolation of the data between MCNP and ANSYS

A complete coupling has been performed admitting to use the neutronic outcomes for thermal-hydraulic and thermo-mechanical calculations.

\section{CONCLUSION}

- At theoretical-computational research campaign has been performed by KIT to create an integrated multiphysics approach to be used for the identification and analysis of BB design points.

7. A test model has been realised accomplishing the complete coupling between the software.

a The correctness and applicability of the approach have been demonstrated, and the great advantages derived from using the same geometry for all the analyses involved in the design of the BB have been also introduced.

$\star^{\star \star} \star$
$\star^{\star} \star \star^{\star}$

